\title{
Smart Vehicle Technology Based on ITS
}

\author{
Cheng Wenming \\ Automotive Engineering institute, Jiangxi University of Technology, Nanchang 330098, China
}

Keywords: Smart transportation system; Smart vehicles; Traffic system; Design of model

\begin{abstract}
The rapid development and wide application of smart traffic system can not only effectively solve the congested traffic, but it can also improve traffic safety, accelerate the treatment and rescue of traffic accidents, promote transportation management and traffic capacity of passengers and freights, reduce environmental pollution and so on. In the paper, it analyzes development status quo of various kinds of smart vehicles, trying to establish a smart vehicle technology based on ITS.
\end{abstract}

\section{Introduction}

The smart traffic system is to combine advanced information technology, data communication transmission technology, electronic control technology as well as computer processing technology and administrative means all together so that it can be effectively applied to service, control and management of traffic transportation comprehensively. Its aim is to make a sound cooperation among people, vehicles and roads in harmony, which can greatly promote comprehensive traffic transportation efficiency, guarantee traffic safety, improve environment quality and promote energy utility rate.

The rapid development and wide application of smart traffic system can not only effectively solve the congested traffic, but it can also have great effect on improving traffic safety, accelerating the treatment and rescue of traffic accidents, promoting transportation management and traffic capacity of passengers and freights, reducing environmental pollution and so on. Therefore, smart traffic system, as an important traffic transportation system in the 21 st century, has been widely valued around the world.

Since the end of 1980s, smart traffic system has been developed consecutively in West Europe, North America and Japan with the establishment of many institutes as well as formulation and implementation of development plan. Such as American smart transportation system association, traffic information and control organization of European Community, Japanese road smart traffic association as well as international standard institute of smart transportation system and so on.

Besides Europe, America and Japan, newly emerged industrial countries and developing countries have also begun developing and studying smart transportation system comprehensively. For example, a comprehensive framework structure and development plan of smart transportation system has been formulated by Ministry of Communications in South Korea on the whole; Singapore has carried out electronic toll collection without parking across the country; and some countries in the Middle East have also begun discussing their research plan for smart transportation system.

Since the beginning of 1980s, China has begun dealing with urban transportation, developing traffic transportation system with the employment of high-end technology. In the middle stage of 1980s, urban traffic control technology has been studied with an extensive urban investigation, 
planning and treatment. At the beginning of 1990s, some colleges and universities as well as traffic research institutes started to study urban traffic induction system technology. These studies mainly learned advanced control systems such as TRANSYT, CSOOT and CSATS from Britain, America and Australia together with advanced theories and thoughts, and meanwhile pilot implementation was carried out in Beijing, Shanghai and Nanjing. At the same time, control systems such as SCOOT or SCATS were introduced to Beijing, Shanghai and Shenyang from Britain and Australia consecutively. These studies and practices developed new fields for China's traffic research, and they have also relieved local traffic contradictions to some extent. However, due to the large differences between China and developed countries regarding characteristics of traffic flow (complicated traffic), road conditions and civilian awareness of traffic laws, the effect of these advanced traffic control and induction systems can't be given full play.

The frame system of ITS mainly manifests in the following aspects. First, we should determine the overall demand of China's ITS; second, we should define ITS as well as its overal systematic framework and structure, logic structure and physical structure; third, we should analyze possible standard issues in ITS system with determination of TJS standard fields; fourth, we should analyze the effect of various transportation meas in the overall ITS system, determining the transportation means and coordination means of administrative departments; at last, we should conduct the planning, implementation as well as economic and technological estimation of ITS. At present, ITS systematic frame and structure has been applied to nine fields, that is, general technical platform, communication information, vehicles, transportation management, traffic management and planning, electronic toll collection (transaction), emergency and safety, comprehensive transportation (hub), economic and technical evaluation.

At present, research on smart vehicles mainly manifests in the following aspects.

(1)Analysis of drivers' behaviors

(2)Perception of environment

(3)Autonomous driving under extreme conditions

(4)Motion control system of vehicles

(5)Active safety system

Research on smart vehicles is related to computer measurement and control, computer vision, sensor data fusion, vehicles engineering and so on. Basically, research on smart vehicles is the combination of computer vision and computer control on vehicles engineering.

Since a smart vehicle system with practical application value should possess timeliness, robustness and practicability, problems on volume and price of computers and CCD should be firstly solved to make vehicle-mounted computer visual navigation system possible, that is, computers should possess stronger and stronger computing capability with the smaller and smaller volume; and the price accounts for a small proportion compared to the overall price of the vehicle. Likewise, CCD and image card have strong image acquisition speed and image pre-treatment with low price. (The timeliness means that the systematic data processing should be synchronous with vehicles' high-speed driving; robustness means that smart vehicles have sound adaptation to different roads such as expressway, city standard road and common road as well as complicated road conditions such as road width, color, texture, dynamic random barriers and traffic flow; and also they have sound adaptation to changing climatic conditions such as sunlight, scene shadows, dusk and night, cloudy, rainy and snowy weather. The practicability means that the cost and volume of smart vehicles can be accepted by common vehicle users.) 


\section{Smart vehicle technology}

Smart vehicles are devoted to promoting automobile's safety and comfort, providing sound interface between vehicles and drivers. Now it is an important part of smart traffic system in all countries, and meanwhile it is a hot issue for world vehicles engineering research as well as a new impetus of automobile industrial growth. Smart vehicles technology can be divided into three layers by functions, which are intelligent perception, pre-warning system, vehicles driving system and autonomous operation system.

Smart perception is to acquire perception for vehicles, vehicles' driving surroundings as well as drivers' state with the employment of various sensors, and if necessary, it can launch replacement information. It mainly includes collision prewarning system and driver's state monitoring system. The collision prewarning system can be further divided into collision warning, blind spot warning, lane departure warning, warning for changing track or combining track, crossroad warning, inspection and warning of passengers, rear collision warning.

Vehicles driving system includes driver's monitoring system and assistant driving system.

The driver's monitoring system mainly includes driver's nap warning system, driver's position occupation monitoring system and so on. The assistant driving system is to decide, plan and propose suggestions with the intelligent perception system information, or it can replace the driver to conduct vehicles' control partially. It mainly includes cruise control, vehicles tracking system, accurate parking system as well as concise maneuvering system.

This is the highest level of smart vehicles technology, which realizes vehicles' operational function completely by vehicle-mounted computer. At present, it has mainly developed the low-speed automatic driving system, autonomous driving system, short-distance vehicles line driving system for congested traffic.

\section{Navigation and positioning technology of smart vehicles}

During the work of smart traffic system, the key issue is to know about the vehicle's position accurately. The traffic management system, dynamic route induction system, automatic charging system and automatic avoidance system of TIS as well as vehicles' height and management system during public transportation rely on vehicles' position information to different extents. If vehicle-mounted navigation and positioning system can't provide TIS with required accuracy and reliability, then most systematic functions of TIS can't be realized. Thus, it is of vital importance to study vehicles' navigation and positioning technology during the application of ITS.

The positioning technology of vehicles can be divided into two kinds. One is to determine vehicle's coordinate position in a certain space by adopting GPS, dead reckoning and so on; the other is to determine the vehicle's position on roads or crossroads. The latter method should rely on maps. At present, vehicle automatic navigation and positioning system can be classified into four varieties, which are autonomous navigation system, administrative highly demand system, road traffic information consultancy system, and road characteristic information collection system respectively. The four kinds of systems are different from each other with mutual relations. As for drivers, their major task is to find a fastest and nearest driving route among the traffic road network. In the long term, drivers mainly choose driving route according to road traffic condition by relying on maps, road traffic signals and past experience. Therefore, autonomous navigation system has achieved wide attention. 
ehicle positioning is the key of navigation system. According to current scientific development level, current vehicle navigation and positioning technology worldwide can be classified into the following types.

1) The global positioning system, which is short for GPS, determines the position of vehicles by receiving signals from four satellites.

2)Global navigation satellite system, which is researched and developed by former Soviet Union, has been further perfected by Russia, known as GNSS.

3)The Inertia Navigation System is made of accelerometer and gyroscope. Generally, it includes three accelerometers and three gyroscopes with high accuracy and strong capability of acquiring data. However, its accuracy will be weak after a long-time work. Therefore, subsidiary sensors are needed such as GPS, etc.

4) Dead reckoning includes electronic compass, rate gyro and speedometer. These sensors can measure driving vehicles' displacement, speed and driving direction. In a short time, such kind of method is of high accuracy, but it may result in accumulative errors after a long-time work.

Map matching is a relative technology determining a vehicle's location matching in a map with street name and address name, which is similar to artificial smart software technology. The vehicle's driving route is related to road network geography. It determines the vehicle's position by a certain historical coordinate such as crossroad. Usually it is combined with other technologies. For example, it corrects the vehicle's positioning errors as well as vehicle's position in municipality with the help of digital road maps.

The TRF system determines the accurate position of vehicle by receiving wireless ratio frequency signals from signal beacons in certain areas with TRF technology. The interactive effect of signals from different positions decides the concise position of vehicles.

7)Signal poles

It includes infrared ray, microwave and $\mathrm{F}$ instrument equipped on the street, usually on the traffic signal station. These signal stations or signal poles can transmit and receive vehicle signals, transmission signals, including traffic information, map data blocks as well as required data for position initialization.

\section{Summary}

The functions of TIS possess autonomous information sensation, task analysis and planning, route planning, route tracking, avoidance, and motion decision and so on artificial intelligence similar to human's smart behaviors besides common functions possessed by other advanced vehicles. The functions above can be realized by the following modules. The modules have formed the models of smart vehicles.

In the paper, it briefly introduces the development situation of smart traffic system. Besides, it analyzes technologies possessed by smart vehicles surrounding demands for smart traffic. It gives a deep study on a series of functions possessed by smart vehicles from visual system, radar system, digital maps, GPS positioning and navigation system, DR dead reckoning system and combined navigation and positioning system so as to meet the need of ITS. 


\section{Acknowledgements}

This work was financially supported by the key subject building project (vehicle engineering) of Jiangxi University of Technology.

\section{References:}

[1] Zimmermann T, Wirtz H, Punal O, et al. Analyzing Metropolitan-Area Networking within Public Transportation Systems for Smart City Applications[C]//New Technologies, Mobility and Security (NTMS), 2014 6th International Conference on. IEEE, 2014: 1-5.

[2] Cheng X, Hu X, Yang L, et al. Electrified vehicles and the smart grid: the ITS perspective[J]. Intelligent Transportation Systems, IEEE Transactions on, 2014, 15(4): 1388-1404.

[3] Charitos D, Theona I, Rizopoulos C, et al. Enhancing citizens' environmental awareness through the use of a mobile and pervasive urban computing system supporting smart transportation[C]//Interactive Mobile Communication Technologies and Learning (IMCL), 2014 International Conference on. IEEE, 2014: 353-358.

[4] Liu P, Zhang S. Some Thoughts About the Future of Intelligent Buildings and Smart Transportation[J]. 2014.

[5] Lee J, Eom J K, Moon D S, et al. A Smart Synthetic Analysis System for Efficient Investment on Transportation Systems in Korea[J]. Procedia Computer Science, 2014, 32: 655-660.

[6] Langer T, Vaidyanathan S. Smart Freight: Applications of Information and Communications Technologies to Freight System Efficiency[M]. Washington, DC: American Council for an Energy-Efficient Economy, 2014.

[7] Lu S, Song H, Xu X. An Enumeration Method Applied in Intelligent Transportation System[J]. International Journal of Smart Home, 2015, 9(2): 143-150.

[8] Liu W, Yan P, Wang Y. Environmental Impact of Electromagnetic Emission of High-speed Maglev Transportation System[C]//CICTP 2014@ sSafe, Smart, and Sustainable Multimodal Transportation Systems. ASCE, 2014: 2782-2787.

[9] Wang F Y. Scanning the Issue and Beyond: Transportation and Mobility Transformation for Smart Cities[J]. Intelligent Transportation Systems, IEEE Transactions on, 2015, 16(2): 525-533.

[10]Chu K K A. Leveraging System Intelligence from Massive Smart Card Database to Support Operational and Strategic Objectives of a Transit Agency[C]//Transportation 2014: Past, Present, Future-2014 Conference and Exhibition of the Transportation Association of Canada//Transport 2014: Du passé vers l'avenir-2014 Congrès et Exposition de'Association des transports du Canada. 2014. 